\section{Comparison of spirometric thresholds in diagnosing smoking-related airflow obstruction: authors' response}

We thank Dr Miller for his comments ${ }^{1}$ on our recent article. ${ }^{2}$ While we agree that age is an important factor in this longstanding controversy, we would like to debate some of the points he makes. First, contrary to his assertions, strong relationships have been shown between CT measures of emphysema and pathological findings. ${ }^{3}$ While there has, in the past, been discussion regarding the 'best' Hounsfield Unit (HU) threshold to define CT emphysema, our use of $-950 \mathrm{HU}$, correlating with more severe disease, has been used widely to demonstrate the power of CT-based density metrics in identifying COPD phenotypes, monitoring longitudinal changes and reducing the population size needed to power pharmaceutical studies. ${ }^{45}$ Second, while we agree with Dr Miller that age may impact the CT-based emphysema score, the effect size is considerably smaller than suggested. Recent data from COPDGene examining normal subjects with a similar age range as our study, with identical scanner protocols, was unable to detect a significant age effect. ${ }^{6}$ Analyses of data from the Multi-Ethnic Study of Atherosclerosis Lung study, which included a much larger and older population, $(n=854)$ showed a small age effect $(0.2 \%$ for every 10 years $)$ on CT-emphysema. ${ }^{7}$ In another large multicentre study, Coxson et $a l^{8}$ noted that the progression of CT-emphysema with advancing age is low. While we agree that future studies should consider age, gender, race and more, we do not believe that the magnitude of the age effect has an influence on our findings. ${ }^{2}$ Third, Dr Miller suggests that our comparisons are not valid, as the discordant Fixed-only group had less emphysema and gas trapping than those positive by both criteria. We believe this relationship is to be expected because those positive by both criteria almost certainly have disease. Most of the argument on which threshold to use is relevant for patients with milder disease in whom spirometric values fall within a narrow margin on either side of defined thresholds, and hence comparisons with 'normal' controls are more illustrative of differences. Contrary to Dr Miller's comments, we did, in fact, adjust for age differences between the Fixedonly and 'normal' patients in multivariate comparisons. Fourth, while we agree that the lower limit of normal (LLN) is a widely used statistical concept, we disagree that there is worldwide acceptance by all scientific disciplines. For example, hypertension and diabetes mellitus are diagnosed on the basis of a fixed threshold above which there is a disproportionate increase in morbidity.

Finally, in addition to quantitative CT, we also examined respiratory morbidity indices and found that the discordant Fixed-only group had worse respiratory quality of life and greater frequency of exacerbations than smoking controls. We stand by our statements that comparisons of long-term outcomes using LLN and Fixed thresholds have shown conflicting results, that LLN has been validated against only the Fixed threshold and that our study is the first head-to-head comparison of LLN with the Fixed threshold using an alternative measure of disease as a gold standard.

We thank Quanjer et al for their comments $^{9}$ on our recent article. ${ }^{2}$ While we agree that CT emphysema and air trapping are not specific for smoking-associated COPD alone, the influence of age on CT-based lung density at total lung capacity is overstated. ${ }^{6} 7810$ We agree that our paper has focused on smokingassociated emphysema. However, age is also a major risk factor for COPD and emphysema, and it can be argued that its effect should not be discounted by adjustments for its impact. ${ }^{11}$ We agree with Quanjer et al that multiple comparisons can introduce type I errors. However, though we did not make a priori adjustments for multiple comparisons, the data presented in the tables are univariate comparisons, the $p$ value for most variables that are significantly different between groups is $<0.001$ and all results point in the same direction, that of the Fixed discordant group having more morbidity, suggesting that it is unlikely these are all type I errors. ${ }^{2}$ We disagree with the assertion that the study grossly misrepresents the COPD population. While we agree that there is growing evidence that nonsmokers are at risk of emphysema and COPD with risk factors starting in early childhood, ${ }^{12}$ smoking (coupled with genetic susceptibility) continues to be considered the single most important risk factor for COPD. We have acknowledged that our comparisons are specifically applicable to this subset of patients with COPD. In fact, the title of our article states that our findings are regarding 'smoking-related airflow obstruction'. We did acknowledge that the use of home oxygen was rather high in the Fixed discordant group and this could be due to comorbidities, high altitude and exertional oxygen desaturation. However, being Fixed discordant appears to identify those at high risk for home oxygen requirement. While we agree with Quanjer et al that the previous literature has been conflicting when comparisons were made between the two thresholds for mortality, neither of the studies cited by Quanjer et al are direct comparisons between the Fixed and LLN thresholds. Also, to overcome the problem of one threshold being used as the gold standard, as was done in previous studies, as well as to overcome the limitation of using only mortality as the outcome, we used respiratory morbidity indices as well as CT presence of disease as gold standard. Data on exacerbations are comparable with other studies that use patient-reported data, and was collected using a rigorous combination of phone calls by research coordinators every 3-6 months using a validated questionnaire and an automated telephony system. ${ }^{13}$ Hence, we believe these data are reasonably robust. While we agree that more research is clearly needed to address this basic question on accurate diagnosis of COPD, we stand by the findings of our study that the Fixed discordant group does represent a subset of patients with clinically significant airflow obstruction and respiratory morbidity. We hope that our study, which provides a link between indirect metrics of lung function to a more direct metric of parenchymal pathology, provides a basis for added insights into what clearly is a continuing controversy.

\section{Surya P Bhatt, ${ }^{1}$ George R Washko, ${ }^{2}$ Mark T Dransfield, ${ }^{1}$ Jessica C Sieren, ${ }^{3}$ John D Newell Jr, ${ }^{3}$ Eric A Hoffman ${ }^{3}$}

${ }^{1}$ Division of Pulmonary, Allergy and Critical Care Medicine, University of Alabama at Birmingham, Birmingham, Alabama, USA

${ }^{2}$ Division of Pulmonary and Critical Care Medicine, Brigham and Women's Hospital, Harvard Medical School, Boston, Massachusetts, USA

${ }^{3}$ Department of Radiology, University of lowa, lowa City, Iowa, USA

Correspondence to Dr Surya P Bhatt, Division of Pulmonary, Allergy and Critical Care Medicine, University of Alabama at Birmingham, THT 422, 1720, 2nd Avenue South, Birmingham, AL 35294, USA; spbhatt@uab.edu

Contributors SPB and EAH drafted the initial response. All authors reviewed the manuscript and added critical information.

Funding COPDGene is supported by NIH Grant Numbers U01 HL089897 and U01 HL089856.

Competing interests MTD received consultancy fees from Boehringer Ingelheim and GlaxoSmithKline. JDN received consultancy fees from VIDA Diagnostics. EAH is the founder of, and owns stocks in, VIDA

Diagnostics. 
Ethics approval IRBs at 21 academic medical centres.

Provenance and peer review Not commissioned; internally peer reviewed.

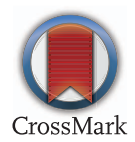

To cite Bhatt SP, Washko GR, Dransfield M T, et al. Thorax 2014;69:1147-1148.

Received 31 July 2014

Revised 19 August 2014

Accepted 21 August 2014

Published Online First 9 September 2014

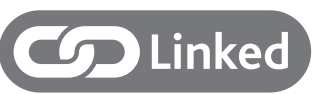

- http://dx.doi.org/10.1136/thoraxjnl-2012-202810

- http://dx.doi.org/10.1136/thoraxjnl-2014-205960

- http://dx.doi.org/10.1136/thoraxjnl-2014-206089

Thorax 2014;69:1147-1148.

doi:10.1136/thoraxjnl-2014-206123

\section{REFERENCES}

1 Miller MR. Comparison of spirometric thresholds in diagnosing smoking-related airflow obstruction. Thorax 2014;69:1145-6.

2 Bhatt SP, Sieren JC, Dransfield MT, et al. Comparison of spirometric thresholds in diagnosing smoking-related airflow obstruction. Thorax 2014;69:409-14.

3 Madani A, Zanen J, de Maertelaer V, et al. Pulmonary emphysema: objective quantification at multi-detector row CT--comparison with macroscopic and microscopic morphometry. Radiology 2006;238:1036-43.

4 Coxson HO, Leipsic J, Parraga G, et al. Using Pulmonary Imaging to Move Chronic Obstructive Pulmonary Disease beyond FEV1. Am I Respir Crit Care Med 2014;190:135-44.

5 Hoffman EA, Simon BA, McLennan G. State of the Art. A structural and functional assessment of the lung via multidetector-row computed tomography: phenotyping chronic obstructive pulmonary disease. Proc Am Thorac Soc 2006;3:519-32.

6 Zach JA, Newell JD Jr, Schroeder J, et al. Quantitative computed tomography of the lungs and airways in healthy nonsmoking adults. Invest Radiol 2012;47:596-602.

7 Hoffman EA, Ahmed FS, Baumhauer $\mathrm{H}$, et al. Variation in the percent of emphysema-like lung in a healthy, nonsmoking multiethnic sample. The
MESA Lung Study. Ann Am Thorac Soc 2014;11: 898-907.

8 Coxson HO, Dirksen A, Edwards LD, et al. The presence and progression of emphysema in COPD as determined by $\mathrm{CT}$ scanning and biomarker expression: a prospective analysis from the ECLIPSE study. Lancet Respir Med 2013;1:129-36.

9 Quanjer PH, Stanojevic S, Thompson BR. Spirometric thresholds and biased interpretation of test results. Thorax 2014;69:1146.

10 Shaker SB, Dirksen A, Ulrik CS, et al. The effect of inhaled corticosteroids on the development of emphysema in smokers assessed by annual computed tomography. COPD 2009;6:104-11.

11 Tuder RM, Kern JA, Miller YE. Senescence in chronic obstructive pulmonary disease. Proc Am Thorac Soc 2012;9:62-3.

12 Lovasi GS, Diez Roux AV, Hoffman EA, et al. Association of environmental tobacco smoke exposure in childhood with early emphysema in adulthood among nonsmokers: the MESA-lung study. Am J Epidemiol 2010;171:54-62.

13 Stewart Jl, Moyle S, Criner GJ, et al. Automated telecommunication to obtain longitudinal follow-up in a multicenter cross-sectional COPD study. COPD 2012;9:466-72. 